Bundesgesundheitsbl 2012 ·55:1361-1362

DOI 10.1007/s00103-012-1561-9

Online publiziert: 21. Oktober 2012

c) Springer-Verlag Berlin Heidelberg 2012

\section{Mielke - L. Schaade}

Robert Koch-Institut, Berlin

\title{
Aufmerksamkeit, Wissen und Verantwortung
}

Liebe Leserinnen, liebe Leser,

das vorliegende und das unmittelbar vorausgehende Heft des Bundesgesundheitsblattes widmen sich dem Schwerpunktthema der nosokomialen und schwierig zu behandelnden Infektionen. In einer Reihe von Beiträgen haben wir uns bemüht, Umfang und Art des Problems transparent zu machen sowie die in Deutschland zur Vermeidung, Erkennung und Bekämpfung etablierten Strukturen, Konzepte und Maßnahmen darzustellen. Dazu konnten in diesen Feldern engagierte Autoren gewonnen werden, denen wir bereits an dieser Stelle für ihre Arbeit und ihren Beitrag ausdrücklich danken möchten.

Im Unterschied zu Erregern klassischer Infektionskrankheiten, die aufgrund besonderer Virulenzeigenschaften durchaus auch beim Gesunden Infektionskrankheiten hervorrufen können, ist das Auftreten nosokomialer Infektionen ganz ausdrücklich durch das Vorliegen disponierender Faktoren, wie z. B. eine intensivmedizinische Behandlung mit ihren invasiven Maßnahmen unter Einsatz von Kathetern und anderen Fremdkörpern, geprägt. Zudem treten diese Infektionen typischerweise in einem Umfeld mit umfangreichem Antibiotikaeinsatz auf, was die Selektion antibiotikaresistenter Bakterien fördert.

„Information und Aufklärung der Allgemeinheit über die Gefahren übertragbarer Krankheiten und die Möglichkeiten zu deren Verhütung" sind ausdrückliches Ziel des Infektionsschutzgesetzes ( $\$ 3$ IfSG). Am Beispiel der nosokomialen Infektionen und der Antibiotikaresistenz wird deutlich, dass die Umset- zung präventiver Maßnahmen den Verantwortungsbereich des von einer Infektion potenziell Bedrohten überschreiten kann und in besonderem Maße das Verantwortungsbewusstsein Dritter im Rahmen pflichtgemäßen Handelns fordert. Der novellierte $\$ 23$ des IfSG verdeutlicht dies ausdrücklich. So ist der Patient darauf angewiesen, dass die Leiter von Krankenhäusern und Einrichtungen für ambulantes Operieren die nach dem Stand des Wissens gebotenen Maßnahmen zur Prävention nosokomialer Infektionen auch tatsächlich umsetzen. Dabei sind nicht alle Risiken in gleichem Maße beherrschbar, und die Einhaltung der Sorgfaltspflicht drückt sich so nicht allein in der Ergebnisqualität (z. B. der Zahl nosokomialer Infektionen und niedrigen Resistenzraten), sondern auch in den zur Risikominimierung etablierten Maßnahmen zur Struktur- und Prozessqualität aus. Die Kommission für Krankenhaushygiene und Infektionsprävention erarbeitet hierzu in einem sorgfältigen Prozess wichtige Empfehlungen und kommt in diesem Heft zur Fortentwicklung ihrer Arbeit zu Wort (Frau Dr. Christiansen und Herr PD Dr. Simon). Um Daten zu nosokomialen Infektionen, zur Antibiotikaresistenz und zum sachgerechten Gebrauch von Antibiotika richtig interpretieren zu können, sind Kenntnisse zu den Methoden des Messens und Vergleichens wichtig. Hierzu nehmen die Beiträge von Frau Prof. Gastmeier und ihrer Mitarbeiterinnen und Mitarbeitern aus dem Nationalen Referenzzentrum für die Surveillance von nosokomialen Infektionen sowie von Herrn Dr. Eckmanns, Frau Dr. Noll und Mitarbeitern, die die am Robert Koch-Institut etablierte Antibiotika-Re-
sistenz-Surveillance in Zusammenarbeit mit engagierten Laboren in Deutschland betreuen, Stellung.

Die im Jahr 2011 auf Initiative des ECDC und mit Unterstützung des Bundesministeriums für Gesundheit und des Robert Koch-Instituts in Deutschland vom NRZ für die Surveillance von nosokomialen Infektionen durchgeführte Punktprävalenzerhebung zu nosokomialen Infektionen und dem Antibiotikagebrauch hat aktuelle Basisdaten für die Einschätzung der Situation geliefert, aus denen sich wichtige Impulse zur notwendigen Verbesserung des Antibiotikaeinsatzes, insbesondere im Zusammenhang mit der perioperativen Prophylaxe ableiten (EpiBull 26/2012). Den teilnehmenden Kliniken soll hier nochmals ausdrücklich gedankt werden.

Die detaillierten Beschreibungen der Ist-Situation und der erkennbaren Trends bei

- MRSA und VRE (NRZ für Staphylokokken und Enterokokken am RKI, Herr PD Dr. Werner und Mitarbeiter/innen),

- gramnegativen Bakterien (NRZ für gramnegative Krankenhauserreger, Dr. Kaase sowie FG13 des RKI, Frau Dr. Pfeifer und C. Eller) sowie

- C. difficile (Konsiliarlabor für C. difficile, PD Dr. von Müller, Dr. Halfmann und Prof. Herrmann)

sind essenzielle Grundlage für die Ableitung rationaler Antibiotikaverordnung, wie sie im Beitrag von Prof. Kern und Dr. de With dargestellt ist (ABS-Fortbildungsinitiative).

Nicht zuletzt durch die Änderungen im IfSG und eine erhöhte Aufmerksam- 
keit werden Häufungen von nosokomialen Infektionen, bei denen ein epidemiologischer Zusammenhang vermutet wird, stärker wahrgenommen. Dies gilt insbesondere in bestimmten Risikobereichen des Krankenhauses, wie z. B. neonatologischen Intensivstationen. Bei der Beherrschung solcher Ereignisse ist ein rasches und koordiniertes Vorgehen von herausragender Bedeutung. In seinem diesbezüglichen Beitrag schildert Prof. Exner seine Erfahrungen im Umgang mit Ausbrüchen auf der Basis der entsprechenden Empfehlung der Kommission für Krankenhaushygiene und Infektionsprävention.

Die Umsetzung strikter Hygienemaßnahmen zur Vermeidung der Weiterverbreitung von Erregern mit besonderen Resistenzen und Multiresistenzen konkurriert je nach betroffenem Bereich mehr oder weniger mit anderen Schutzbzw. Behandlungszielen. Heime für alte Menschen, die den Lebensbereich der betreuten Menschen darstellen und Einrichtungen für die medizinische Rehabilitation mit ihren vielfältigen physiotherapeutischen Anwendungen und Gemeinschaftsaktivitäten sind Beispiele dafür. Zwei Beiträge widmen sich daher ausdrücklich dieser Thematik (Dr. Ruscher et al. zu den Herausforderungen bei alten Menschen in Heimen und Dr. Hergenröder et al. zum Umgang mit MRSA in der medizinischen Rehabilitation).

Grundlage für jedes sachgerechte Handeln zur Erkennung, Vermeidung und Beherrschung von Infektionsrisiken ist in allen Berufsfeldern der Medizin eine fundierte Ausbildung. In einem von der historischen Entwicklung ausgehenden Bogen stellen Prof. Exner und Prof. Kramer die Situation und Bedeutung von Verbesserungen in der Aus-, Fort- und Weiterbildung in der Hygiene und Infektionsprävention dar und komplettieren damit die im Rahmen von DART und der Umsetzung des novellierten IfSG sowie der Krankenhaushygieneverordnungen der Länder als notwendig formulierten Aktivitäten im Bereich der Qualifizierung von Fachpersonal.

Schließlich hat sich das Instrument der regionalen Netzwerke zur Vermeidung der Weiterverbreitung von mehrfachresistenten Bakterien vom Pilotpro- jekt zu einer in Deutschland fest etablierten Struktur gemausert. Im abschließenden Beitrag wird über das inzwischen 3. Treffen der Moderatoren der Netzwerke am Robert Koch-Institut berichtet. Ein weiteres Treffen ist für das Jahr 2013 geplant.

In der Zusammenschau der Beiträge wird deutlich, dass nun ein Kanon wichtiger Instrumente zur Erkennung, Vermeidung und Bekämpfung nosokomialer Infektionen und antibiotikaresistenter Bakterien etabliert ist. Während die Daten zu MRSA Anlass zur Hoffnung geben, machen die Trends bei den gramnegativen Bakterien deutlich, dass die nächsten Jahre von einer noch konsequenteren Umsetzung der empfohlenen sowie der gesetzlich verankerten Maßnahmen geprägt sein müssen. Dabei ist zu beachten, dass das Problem der Antibiotikaresistenz zu seiner Beherrschung eines komplexen und internationalen Ansatzes bedarf. Dies machen auch die Befunde zur Antibiotikaresistenz in der Tiermast und die zum Teil erhebliche Verbreitung von Carbapenemase-bildenden Bakterien in bestimmten Regionen der Welt deutlich. Die Deutsche Antibiotika-Resistenzstrategie DART berücksichtigt diese Aspekte. Wenn dieses Heft zu mehr Aufmerksamkeit und Wissen im Bereich der nosokomialen Infektionen und der Antibiotikaresistenz beiträgt, hätte es unsere damit verknüpften Wünsche und Hoffnungen erfüllt.

Ihre

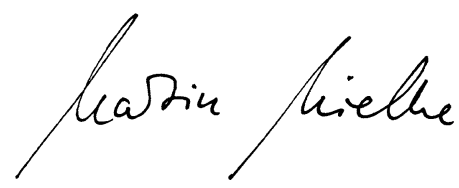

Martin Mielke

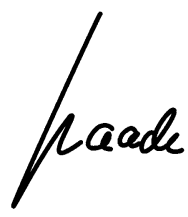

Lars Schaade

\section{Korrespondenzadressen}

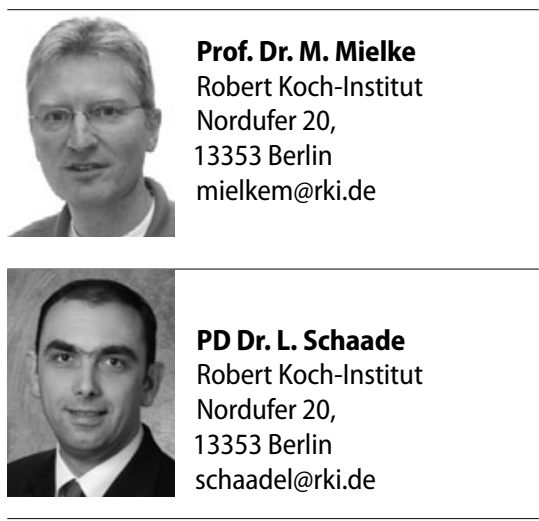

Literatur

1. Mielke M (2010) Prevention and control of nosocomial infections and resistance to antibiotics in Europe - Primum non-nocere: elements of successful prevention and control of healthcare-associated infections. Int J Med Microbiol 300(6):346-350 